\title{
INFLUÊNCIA DA MARCHA SOBRE O ERITROGRAMA EM EQUINOS DA RAÇA MANGALARGA MARCHADOR
}

\author{
Clarisse S Coelho ${ }^{1}$ \\ Vinicius Vaneli Fardin ${ }^{2}$ \\ Gabriella Agra de Omena Silva ${ }^{3}$ \\ Renan Silva Carvalho ${ }^{2}$ \\ Evandro Pereira Neto ${ }^{4}$
}

\begin{abstract}
RESUMO
O objetivo da presente pesquisa foi avaliar a influência do exercício físico (marcha) sobre o eritrograma de equinos da raça Mangalarga Marchador. Para tal, 15 equinos da referida raça, nove fêmeas e seis machos, com 4,6 $\pm 1,9$ anos de idade e pesando 399,0 $\pm 34,0 \mathrm{~kg}$, foram avaliados em quatro diferentes momentos: antes (T0), 5 minutos (T1), 30 minutos (T2) e 120 minutos (T3) após a execução de 40 minutos de marcha. Amostras de sangue foram obtidas para determinar volume globular (VG), número de eritrócitos e concentração de hemoglobina. Posteriormente foram calculados volume corpuscular médio (VCM) e concentração de hemoglobina corpuscular média $(\mathrm{CHCM})$. As variáveis foram analisadas quanto a normalidade pelo teste de Kolmorov-Smirnov, seguido de análise de variância e comparações entre médias pelo teste de Tukey, considerando $\mathrm{p}<0,05$. Os resultados demonstraram que a marcha influenciou de forma significativa VG $(\mathrm{p}=0,0016)$, número de eritrócitos $(\mathrm{p}=0,0013)$ e concentração de hemoglobina $(\mathrm{p}=0,0004)$, com maiores valores registrados em T1. Não foram observadas diferenças para VCM ( $\mathrm{p}=0,8697)$ e CHCM ( $\mathrm{p}=0,4980)$. Foi possível concluir que a marcha no protocolo proposto levou a importantes alterações no eritrograma e tais alterações devem ser bem compreendidas e caracterizadas para seu uso correto na avaliação do condicionamento atlético de equinos marchadores.
\end{abstract}

Palavras-chave: cavalos, eritrócitos, exercício.

\section{INFLUENCE OF MARCHA GAIT ON THE ERYTHROGRAM OF MANGALARGA MARCHADOR HORSES}

\begin{abstract} erythrogram of Mangalarga Marchador. Fifteen Mangalarga Marchador horses, nine females aerobic exercise characterized by 40 minutes of marcha gait. Blood samples were taken to determine packed cell volume (PCV), concentration of erythrocytes and hemoglobin. From these data, mean corpuscular volume (MCV) and mean corpuscular hemoglobin concentration (MCHC) were calculated. Variables were analyzed for normality with Kolmorov-Smirnov $(\mathrm{p}=0.0013)$ and hemoglobin $(\mathrm{p}=0.0004)$, with higher values recorded on T1. No significant

\footnotetext{
${ }^{1}$ Programa de Mestrado em Ciência Animal - Universidade Vila Velha (UVV-ES). Correspondência.

${ }^{2}$ Residente do Setor de Grandes Animais - Hospital Veterinário - UVV-ES.

${ }^{3}$ Graduando Faculdade de Medicina Veterinária e Zootecnia-UVV-ES.

${ }^{4}$ Universidade Federal da Bahia (UFBA).
}

The aim of this study was to evaluate the influence of physical exercise (marcha gait) on the and six males, aged $4.6 \pm 1.9$ years old and weighting $399.0 \pm 34.0 \mathrm{~kg}$, were evaluated in four different moments: before (T0), 5 minutes (T1), 30 minutes (T2) and 120 minutes (T3) after test and comparisons were made using Tukey test, considering $\mathrm{p}<0.05$. Results showed that the imposed exercise altered significantly PCV $(\mathrm{p}=0.0016)$ and concentration of erythrocytes 
differences were observed for hematological indexes MCV $(\mathrm{p}=0.8697)$ and MCHC $(\mathrm{p}=0.4980)$. It was possible to conclude that the physical activity imposed led to important alterations on erythrogram and these modifications have to be well comprehended to be used appropriately on the evaluation of marchador horses.

Keywords: horses, erythrocytes, exercise.

\title{
INFLUENCIA DE LA MARCHA EN EL ERITROGRAMA DE CABALLOS MANGALARA MARCHADOR
}

\begin{abstract}
RESUMEN
El objetivo del presente estudio fue evaluar la influencia del ejercicio físico (marcha) sobre el eritrograma de equinos de la raza Mangalarga Marchador. Para esto, 15 equinos, nueve hembras y seis machos, con 4,6 $\pm 1,9$ anos de edad y pesando $399,0 \pm 34,0 \mathrm{~kg}$, fueron evaluados en cuatro tiempos diferentes: antes (T0), 5 minutos (T1), 30 minutos (T2) y 120 minutos (T3) después de 40 minutos de marcha. Muestras de sangre fueron obtenidas para determinar el volumen globular (VG), número de eritrocitos y concentración de hemoglobina. Posteriormente, fueron calculados el volumen corpuscular medio (VCM) y la concentración de hemoglobina corpuscular media (CHCM). Estas variables fueron analizadas en cuanto a normalidad, por medio del teste de kolmorov-smirnov, seguido de análisis de variancia y comparaciones entre las medias por el teste de Tukey, considerando $p<0,05$. Los resultados demostraron que la marcha influenció de forma significativa el volumen globular $(\mathrm{p}=0,0016)$, número de eritrocitos $(\mathrm{p}=0,0013)$ y concentración de hemoglobina $(\mathrm{p}=0,0004)$, con mayores valores registrados en T1. No fueron observadas diferencias para VCM ( $\mathrm{p}=0,8697)$ у CHCM $(\mathrm{p}=0,4980)$. Fue posible concluir que la marcha, en el protocolo propuesto, produjo importantes alteraciones en el eritrograma.
\end{abstract}

Palabras clave: caballos, eritrocitos, ejercicio.

\section{INTRODUÇÃO}

O exercício físico realizado durante treinamentos ou competições gera em humanos e animais variações em diversos parâmetros fisiológicos (1). A compreensão de tais mecanismos e sua caracterização são essenciais na avaliação da performance e da eficácia de um programa de treinamento / condicionamento (2). Existem diversos estudos na literatura (36) avaliando a performance de cavalos de diversas modalidades atléticas, sendo poucos os relatos envolvendo equinos da raça Mangalarga Marchador, treinados em condições climáticas tropicais. Tipicamente brasileira, os animais da referida raça têm um caminhar característico, a marcha, que representa um teste exaustivo sem similar em todo o mundo, onde o animal realiza um exercício de longa duração, com grande gasto energético, num percurso em círculo, sem repouso e em velocidade constante (7).

Tyler-McGowan et al. (8) citaram que variáveis hematimétricas, tais como volume globular (VG), contagem de eritrócitos e concentração de hemoglobina, podem ser usadas na avaliação do exercício e do treinamento físico. Segundo Manohar et al. (9), aumentos nos valores hematimétricos ocorrem de acordo com a intensidade do exercício e se devem à hemoconcentração e/ou à contração esplênica, tendendo a normalidade 45 minutos após a atividade física. Esse efeito fisiológico é vantajoso, particularmente nos equinos atletas, pois eleva a capacidade aeróbica ao aumentar a quantidade de eritrócitos na circulação (10) e aumentar o fluxo de sangue da microcirculação muscular (11). Exercícios de intensidade 
submáxima geram menor atividade simpática, e consequentemente uma contração esplênica incompleta, em relação aos exercícios de alta intensidade (12).

Kowal et al. (4) trabalharam com equinos da raça Puro Sangue Inglês (PSI), submetidos a teste de esforço em esteira, e encontraram aumentos significativos no VG, contagem de eritrócitos e concentração de hemoglobina relacionados com o incremento do esforço físico. Evans (13) cita que o VG aumenta linearmente com o aumento da intensidade do exercício, podendo atingir $61 \%$ em PSI. Em pesquisa realizada com 24 equinos treinados da raça Puro Sangue Árabe (PSA) submetidos ao teste de esforço crescente em esteira ergométrica, Ferraz et al. (5) descreveram variações significativas a partir do repouso para todas as variáveis hematimétricas, relacionando tais achados com a contração esplênica esforço-dependente. Tais resultados foram semelhantes aos encontrados por Machado (14) que estudou o metabolismo oxidativo eritrocitário em equinos da raça Árabe submetidos a exercício em esteira.

Avaliando equinos mestiços Quarto de Milha submetidos à prova de team penning, Miranda et al. (11) observaram aumentos significativos para hemoglobina, hemácias e VG, relacionando tais achados a uma associação entre a perda de líquidos pela sudorese, troca transitória de fluidos entre compartimento extra e intravascular e contração esplênica.

Apesar de vários trabalhos estudando os efeitos do exercício físico sobre as diversas variáveis hematimétricas, Miranda et al. (11) citam que os parâmetros hematológicos são influenciados pela raça, idade, sexo, alimentação e exercício físico e daí a importância de avaliar equinos nas diferentes modalidades atléticas. As informações são escassas em relação a equinos da raça Mangalarga Marchador e, sendo assim, o objetivo da pesquisa foi avaliar a influência do exercício físico (marcha) sobre o eritrograma em equinos da raça Mangalarga Marchador criados no Estado do Espírito Santo. A hipótese dos autores é que ocorram modificações e as mesmas devem ser bem caracterizadas para auxiliar de forma correta a avaliação de performance atlética.

\section{MATERIAL E MÉTODOS}

O presente projeto de pesquisa teve aprovação do Comitê de Bioética Animal da Universidade Vila Velha (CEUA - UVV-ES), sendo registrado sob o número 72/2009, aprovado em 25 de fevereiro de 2010.

Foram utilizados 15 equinos da raça Mangalarga Marchador, sendo nove fêmeas e seis machos, pesando em média $399 \pm 34 \mathrm{~kg}$, com idade variando entre três e dez anos (média de 4,6 $6 \pm 1,9$ anos de idade), considerados clinicamente hígidos, mediante exames físicos e laboratorial (hemograma) (15). Estes animais pertencem a dois haras de criadores de equinos Mangalarga Marchador, localizados na região de Vitória - ES, Brasil.

Todos os animais eram submetidos ao mesmo tipo de manejo alimentar (16) e sanitário. A dieta dos animais foi feita com feno de coast-cross (Cynodon dactylon) e sal mineral inorgânico (Essencefós, Nutrimentos Presence, Paulínia, SP, Brasil) ad libitum e ração comercial $(1,2 \mathrm{~kg} / 100 \mathrm{~kg}$ de peso corporal - Corcel Tradicional, Nutrimentos Presence, Paulínia, SP, Brasil) com 12\% proteína bruta (3500 kcal de energia), divididas em três porções diárias. A água foi fornecida ad libitum.

Todos os equinos selecionados encontravam-se no mesmo estágio de treinamento e executavam tal atividade há pelo menos seis meses. $O$ treinamento semanal consistia de exercício com o animal ao passo sendo puxado pelo cabresto por um período médio de 60 minutos, duas vezes na semana, alternando nos demais três dias em exercícios montados (marcha), por 40 minutos. Aos finais de semana, os animais marchavam por 20 minutos em cada dia. 
Os animais foram submetidos a um teste físico a campo, no qual executaram marcha batida, sendo 20 minutos em sentido horário e 20 minutos em sentido anti-horário, em protocolo similar ao recomendado pela Associação Brasileira de Criadores de Cavalos da raça Mangalarga Marchador (ABCCMM). Todas as atividades foram realizadas no período da manhã (entre 6h:00 e 11h:00), quando também foram registradas as características da pista. Foram usados dois cavaleiros com peso corpóreo aproximado de $70 \mathrm{~kg}$ e altura de $1,70 \mathrm{~m}$.

Cada cavalo foi avaliado antes do exercício físico, com o animal ainda na baia (T0), logo após o término da atividade física, com o animal ainda na pista (T1) e com 30 minutos (T2) e 2 horas de recuperação (T3). Amostras de sangue foram obtidas, após antissepsia local, por meio de venopunção da jugular com agulhas descartáveis (25 $\mathrm{mm}$ x $0,8 \mathrm{~mm})$, utilizandose sistema a pressão negativa, em tubos de vidro contendo anticoagulante EDTA com capacidade de $4 \mathrm{~mL}$, para determinação do eritrograma. Todas as 60 amostras foram transportadas sob refrigeração ao Laboratório Clínico do Centro de Diagnóstico Veterinário (CDV, Vitória-ES), onde foram imediatamente processadas.

Contagem de eritrócitos, concentração de hemoglobina e volume globular, bem como cálculos dos índices hematimétricos (VCM - volume corpuscular médio e CHCM concentração de hemoglobina corpuscular média) foram feitos em analisador hematológico (17) (Coulter HmX Hematology Analyzer, Beckman Coulter, Brea, CA, USA).

Adicionalmente, durante o exercício, os equinos usaram um monitor cardíaco com GPS (RS800CX-G3, Polar Electro, Lake Success, NY, EUA) para registro da velocidade e distância percorrida por cada animal. Os dados foram analisados usando o programa ProTrainer (Polar Electro, Lake Success, NY, EUA).

A análise dos resultados foi realizada utilizando-se o programa estatístico computadorizado GraphPad InStat, versão 3.0 (GraphPad, La Jolla, CA, USA) e os eesultados foram expressos na forma de média \pm desvio-padrão. Os dados foram avaliados quanto a normalidade pelo teste de Kolmogorov-Smirnov, seguido de análise de variância de medidas repetidas (ANOVA) e comparação entre médias (teste de Tukey). Nestas análises levou-se em consideração a influência do exercício físico (marcha) sobre as concentrações das variáveis estudadas. Valores de $\mathrm{p} \leq 0,05$ foram considerados significativos.

\section{RESULTADOS E DISCUSSÃO}

Nos exames clínicos realizados para seleção dos equinos usados na presente pesquisa, as médias registradas foram: frequência cardíaca de $45 \mathrm{bpm}$, frequência respiratória de 20 mpm, motilidade intestinal presente à auscultação, mucosas róseas e temperatura retal de $37,5^{\circ} \mathrm{C}$. Na avaliação do hemograma, os valores médios encontrados foram de 7,25 x10\% $/ \mu \mathrm{L}$ para número de eritrócitos, 11,99 g/dL para concentração de hemoglobina, 35\% para volume globular, 49,01 fl para VCM (volume corpuscular médio), 33,79 g/dL para CHCM (concentração de hemoglobina corpuscular média) e 9533 leucócitos/ $\mu \mathrm{L}$. Tais valores encontram-se dentro da normalidade segundo Robinson (18), com todos os animais selecionados registrando valores dentro da faixa de normalidade.

Os equinos usados foram exercitados em três dias, sendo cinco animais em cada dia. As características do clima local são típicas de regiões tropicais, com altas temperaturas e alta umidade. A temperatura média local nos três dias foi de $24 \mathrm{oC}$ e umidade relativa do ar de $80 \%$. A pista de grama oval de aproximadamente $130 \mathrm{~m}$ encontrava-se seca. Segundo os cavaleiros, os animais responderam bem a atividade física executada, não demonstrando desconforto durante a realização da mesma. Os animais atingiram velocidade média de $11,3 \pm 0,5 \mathrm{~km} / \mathrm{h}$ e uma distância média de $7,2 \pm 1,1 \mathrm{~km}$ durante os 40 minutos de exercício, similar a descrição de Rezende (7). 
Na Tabela 1 estão apresentados os valores médios e desvios-padrão para os valores do eritrograma, além dos valores de $\mathrm{p}$ obtidos na análise de variância (ANOVA). É possível observar que houve aumento significativo do $\mathrm{VG}(\mathrm{p}=0,0016)$, do número de eritrócitos $(\mathrm{p}=0,0013)$ e da concentração de hemoglobina $(\mathrm{p}=0,0004)$, com maiores valores registrados em T1. Não foram observadas diferenças para VCM ( $\mathrm{p}=0,8697)$ e CHCM $(\mathrm{p}=0,4980)$.

Tabela 1. Resultados do eritrograma nos equinos da raça Mangalarga Marchador avaliados durante uma sessão de exercício físico (marcha). Vila Velha, 2016.

\begin{tabular}{|l|c|c|c|c|c|}
\hline & T0 & T1 & T2 & T3 & $p$ \\
\hline $\mathrm{VG}(\%)$ & $35,5 \pm 4,6^{\mathrm{a} *}$ & $40,6 \pm 4,9^{\mathrm{b}}$ & $34,8 \pm 4,2^{\mathrm{a}}$ & $35,8 \pm 3,3^{\mathrm{a}}$ & 0,0016 \\
\hline $\mathrm{He}\left(\mathrm{x} 10^{6} / \mu \mathrm{L}\right)$ & $7,3 \pm 0,9^{\mathrm{a}}$ & $8,5 \pm 1,1^{\mathrm{b}}$ & $7,1 \pm 1,2^{\mathrm{a}}$ & $7,3 \pm 0,8^{\mathrm{a}}$ & 0,0013 \\
\hline $\mathrm{Hb}(\mathrm{g} / \mathrm{dL})$ & $12,0 \pm 1,5^{\mathrm{a}}$ & $14,0 \pm 1,7^{\mathrm{b}}$ & $11,7 \pm 1,7^{\mathrm{a}}$ & $12,0 \pm 1,3^{\mathrm{a}}$ & 0,0004 \\
\hline $\mathrm{VCM}(\mathrm{fl})$ & $49,0 \pm 3,6^{\mathrm{a}}$ & $48,3 \pm 3,8^{\mathrm{a}}$ & $49,0 \pm 4,9^{\mathrm{a}}$ & $49,6 \pm 4,0^{\mathrm{a}}$ & 0,8697 \\
\hline $\mathrm{CHCM}(\mathrm{g} / \mathrm{dL})$ & $33,8 \pm 1,7^{\mathrm{a}}$ & $34,5 \pm 1,5^{\mathrm{a}}$ & $33,7 \pm 2,1^{\mathrm{a}}$ & $33,6 \pm 1,5^{\mathrm{a}}$ & 0,4980 \\
\hline
\end{tabular}

* Letras minúsculas diferentes na mesma linha denotam diferença significativa entre as médias (p < 0,05$)$ obtido pelo teste de Tukey. VG: volume globular, He: contagem de eritrócitos, Hb: concentração de hemoglobina, VCM: volume corpuscular médio, CHCM: concentração de hemoglobina corpuscular média. T0: antes da atividade física, T1: imediatamente após o término da atividade física, T2: com 30 minutos de recuperação, T3: com 2 horas de recuperação.

Segundo Ferraz et al. (5) e Kowal et al. (4), o aumento das variáveis hematimétricas, conforme também observado para os equinos da raça Mangalarga Marchador usados na presente pesquisa para VG, contagem de eritrócitos e concentração de hemoglobina, estaria relacionada a uma contração esplênica esforço-dependente, proporcional à atividade física imposta. Segundo Persson (10), o sistema nervoso simpático é o responsável pela mobilização esplênica, após sensibilização dos receptores adrenérgicos alfa. Isso é extremamente benéfico para os animais, visto que há aumento no número de eritrócitos e da hemoglobinemia, aumentando, portanto, a capacidade de transporte de oxigênio para o músculo esquelético $(11,19)$. Tais achados também foram descritos por Garcia et al. (3), estudando 17 equinos da raça Crioulo antes e sequencialmente após um período de treinamento. Os autores justificaram os aumentos na concentração de hemoglobina e do VG também como decorrentes da contração esplênica.

De acordo com Jain (20), a contração esplênica que ocorre no exercício físico introduz na circulação periférica uma grande quantidade de eritrócitos que podem aumentar em $40 \% \mathrm{o}$ VG. Entretanto, a hemoconcentração também ocorreria devido a diminuição do volume plasmático, devido a perdas pela sudorese e respiração e a redistribuição do volume vascular. Conforme supracitado, Miranda et al. (11) citam que os parâmetros hematológicos precisam ser estudados nos equinos nas diferentes modalidades atléticas. Na presente pesquisa, o VG registrado imediatamente após a execução da marcha foi inferior aos descritos em equinos da raça Árabe $(5,14)$, em equinos da raça Crioulo (3) e em equinos Puro Sangue Inglês (PSI) (4), com os últimos registrando valores de $65,7 \%$. Miranda et al. (11) registraram valores semelhantes aos da presente pesquisa nos equinos da raça Quarto de Milha usados em provas de team penning. Acredita-se que o PSI tenha uma maior reserva esplênica que as demais raças e equinos usados em provas de resistência, tais como Árabe e Mangalarga Marchador, teriam um maior volume plasmático (5), fato esse que poderia justificar as diferenças entre as raças.

Conforme já descrito, não houve diferença significativa para VCM e CHCM, sugerindo que a marcha não gerou alteração no tamanho dos eritrócitos e/ou aumento na concentração de hemoglobina dos mesmos, semelhante a Balarin et al. (19), estudando equinos da raça Puro Sangue Inglês em treinamento na pista, e Machado (14), trabalhando com equinos da raça Árabe submetidos a esforço progressivo em esteira rolante. Segundo Machado (14), o VCM não se alterou porque dois eventos se sobrepõem, a liberação de células velhas menores por 
parte do baço e a tumefação dos eritrócitos em decorrência da mobilização de líquidos e maior concentração eritrocitária de íons no controle da acidose metabólica devido ao exercício, dados que também podem ser sugeridos para justificar os achados na presente pesquisa. A determinação da distribuição do diâmetro dos eritrócitos (RDW), feita por Machado (14) e Balarin et al. (19), confirmou tal teoria ao demonstrar uma heterogeneidade no pós-exercício, fato não demonstrado pelo VCM.

Diferentemente, Ferraz et al. (5) observaram aumento de VCM e CHCM na etapa de esforço máximo e sugeriram que as diferenças com outras pesquisas pode estar relacionada com intensidade do esforço físico ou com as diferenças que podem existir nos testes a campo e nos testes com esteira. Com base nisso, torna-se importante considerar como os testes foram executados a fim de permitir a comparação e discussão dos resultados. Em se tratando de pesquisas a campo, coletar em intervalos seriados durante a execução do exercício, associando a um incremento de esforço, é extremamente difícil, levando a constantes processos de aceleração e desaceleração que poderiam influenciar os resultados. Evans et al. (21) descreveram que aferição da frequência cardíaca (FC), lactato sanguíneo, contagem de eritrócitos e concentração de hemoglobina, determinadas antes e após a execução do exercício, são índices confiáveis de avaliação das adaptações fisiológicas de cavalos atletas. Segundo Balarin et al. (19), as coletas após o exercício devem ser realizadas em até 5 minutos após o termino da atividade física, semelhante ao executado na presente pesquisa, conotando que nesse momento os equinos trabalhados ainda não entraram em desaceleração. A partir desse momento, as variáveis hematimétricas de uma forma geral tendem a diminuir, conforme também observaram Ferraz et al. (5), inclusive para VCM e CHCM.

Kowal et al. (4) citaram que a normalização das variáveis hematimétricas tende a acontecer 45 minutos após a atividade física, fato comprovado na presente pesquisa, quando VG, concentração de hemoglobina e contagem de eritrócitos atingiram, aos 30 minutos após a execução da marcha, os valores registrados previamente ao teste.

\section{CONCLUSÕES}

A análise dos resultados permite concluir que nos equinos da raça Mangalarga Marchador, fisicamente bem condicionados usados na presente pesquisa, o exercício físico de 40 minutos de marcha levou a uma aumento significativo dos valores de VG, contagem de eritrócitos e concentração de hemoglobina, cujos valores retornaram aos valores basais dentro de 30 minutos após a suspensão da atividade. Tais alterações devem ser bem compreendidas e caracterizadas para seu uso correto na avaliação do condicionamento atlético de equinos marchadores.

\section{AGRADECIMENTOS}

À UVV-ES, pelo financiamento do projeto. Suporte Financeiro: UVV-ES

\section{REFERÊNCIAS}

1. Kienzle E, Freismuth A, Reusch A. Double blind placebo controlled vitamin E ou selenium supplementation of Sport horses with unspecified muscle problems. J Nut. 2006;136:2045-7.

2. Marques MS, Fernandes WR, Coelho CS, Mirandola R. Influência do exercício físico sobre os níveis de lactato plasmático e cortisol sérico em cavalos de corrida. Hora Vet. 2002;22:29-32. 
3. Garcia M, Guzman R, Cabezas I, Merino V, Palma C, Pérez R. Evaluación del entrenamiento tradicional del caballo criollo chileno de rodeo mediante el análisis de variables. Arch Med Vet. 1999;31:167-76.

4. Kowal RJ, Almosny NRP, Cascardo B, Summa RP, Cury LJ. Avaliação dos valores hematológicos em cavalos (Equus caballus) da raça Puro-Sangue-Inglês submetidos a teste de esforço em esteira ergométrica. Rev Bras Cienc Vet. 2006;13:25-31.

5. Ferraz GC, Teixeira Neto AR, D'Angelis FHF, Lacerda Neto JC, Queiroz Neto A. Alterações hematológicas e cardíacas em cavalos árabes submetidos ao teste de esforço crescente em esteira rolante. Braz J Vet Res Anim Sci. 2009;46:431-7.

6. Pritchard JC, Burn CC, Barr ARS, Whay HR. Haematological and serum biochemical reference values for apparently healthy working horses in Pakistan. Res Vet Sci. 2009;87:389-95.

7. Rezende ASC. Aditivos ou suplementos? Mangalarga marchador. Rev Of ABCCMM. 2006;18:44-8.

8. Tyler-McGowan CM, Golland LC, Evans DL, Hodgson DR, Rose RJ. Haematological and biochemical responses to training and overtraining. Equine Vet J. 1999;30:621-5.

9. Manohar M, Goetz TE, Hassan AS. Effect of prior high intensity exercise-induced arterial hypoxemia in thoroughbred horses. J Appl Phys. 2001;90:2371-7.

10. Persson S. On blood volume and working capacity in horses. Studies of methodology and physiological and pathological variations. Acta Vet Scand. 1967;19:189.

11. Miranda RL, Mundim AV, Saquy ACS, Costa AS, Guimarães EC, Gonçalves FC, et al. Perfil hematológico de equinos submetidos à prova de Team Penning. Pesqui Vet Bras. 2011;31:81-6.

12. Lekeux P, Art T, Linden A, Desmecht D, Amory H. Heart rate, hematological and serum biochemical responses to show jumping. In: Persson SGB, Lindholm A, Jeffcott LB. Equine exercise physiology. Davis: ICEEP Publications; 1991. p.385-90.

13. Evans DL. Overview of equine exercise physiology and biochemistry. In: Evans DL. Training and fitness in athletic horses. Sydney: Rural Industries Research and Development Corporation; 2000. p.10-32.

14. Machado LP. Eritrograma, glutationa reduzida e superóxido dismutase eritrocitários e metahemoglobina em equinos da raça Árabe submetidos a exercício em esteira. Efeito da suplementação com vitamina E (DL-Alfa-tocoferol) [dissertação]. Botucatu: Faculdade de Medicina Veterinária e Zootecnia, Universidade Estadual Paulista; 2011.

15. Speirs CV. Clinical examination of horses. Pennsilvania: Saunders; 1997.

16. Ralston SL. Equine clinical nutrition: specific problems and solutions. Comp Cont Educ. 1988;10:356-63. 
17. Kramer JW. Normal hematology of the horse. In: Feldman BF, Zinkl JG, Jain CN. Schalm's veterinary hematology. 5th ed. Philadelphia: Lippincott Williams; 2000. p.1069-74.

18. Robinson EN. Current therapy in equine medicine. 5th ed. Philadelphia: Saunders; 2003.

19. Balarin MRS, Lopes RS, Kohayagawa A, Laposy CB, Fonteque JH. Valores da amplitude de distribuição do tamanho dos eritrócitos (RDW) em eqüinos Puro Sangue Inglês (PSI) submetidos a exercícios de diferentes intensidades. Braz J Vet Res Anim Sci. 2006;43:637-41.

20. Jain NC. Examination of the blood and bone marrow. In: Jain NC. Essentials of veterinary hematology. Philadelphia: Lea and Febiger; 1993. p.1-18.

21. Evans DL, Harris RC, Snow DH. Correlation of racing performance with blood lactate and heart rate after exercise in Thoroughbred horses. Equine Vet J. 1993;25:441-5.

Recebido em: 19/08/2016

Aceito em: 28/07/2017 